Thorax (1963), 18, 54.

\title{
Changes in calibre of the smaller airways in man
}

\author{
ROBERT MARSHALL ANDW. S. HOLDEN \\ From the Nuffield Department of Surgery, Radcliffe Infirmary, Oxford, and the Department of \\ Radiodiagnosis, United Oxford Hospitals
}

The changes in calibre which occur in the trachea and large bronchi during respiration and coughing have been observed by bronchoscopy and measured by bronchography (Dekker and Groen, 1957 ; Holden and Ardran, 1957 ; Ross, Gramiak, and Rahn, 1955; Stutz, 1950a, b). The small airways of less than $3 \mathrm{~mm}$. diameter appear to be responsible for about $90 \%$ of the resistance of the bronchial tree during normal respiration (Rohrer, 1915). The present study was undertaken to observe particularly the changes in the smaller airways, including the smaller bronchi and bronchioli, at varying lung volumes and during coughing.

\section{METHODS}

The subjects investigated were patients who had had bronchograms for routine diagnostic purposes and in whom the region of lung investigated in this study was considered by bronchography to be within normal limits. Bronchograms were carried out by the injection of Dionosil Oily (Glaxo) through the cricothyroid membrane under local anaesthesia and without premedication. The bronchial diameters were measured from magnified films taken with a tube-tocouch distance of $43 \mathrm{~cm}$. and a tube-to-film distance of $86 \mathrm{~cm}$. The focal diameter of the anode was $0.3 \mathrm{~mm}$. Exposures were made at $120 \mathrm{kVp}, 20 \mathrm{~mA}$. for 0.02 to 0.04 second, using fast films and screens.

By this method bronchi which lie $10 \mathrm{~cm}$. above the couch, i.e., about the centre of the lung in a normal person, are magnified 2.6 times on the film. Bronchi near the front of the chest, say $16 \mathrm{~cm}$. from the couch, are magnified 3.2 times, and the posterior bronchi, say $4 \mathrm{~cm}$. from the couch, are magnified 2.2 times. This magnification method has the advantage that the bronchi of less than $1 \mathrm{~mm}$. diameter can be measured directly from the film. The method suffers from the disadvantage that movements of the bronchi during inspiration or expiration in an antero-posterior direction will give a change in magnification. The greatest change in antero-posterior position would be expected to occur in the bronchi close to the anterior chest wall and the least change in the posterior bronchi which move only a little relative to the 1 Present address: Torbay Hospital, Torquay, S. Devon. couch. During a maximal inspiration the sternum moves about $3 \mathrm{~cm}$. anteriorly in an adult male with a vital capacity of 5 litres. This would increase the magnification of the most anterior bronchi from 3.2 to 3.6 times, a maximum increase of $12 \%$. Most of the bronchi measured in these studies were lateral bronchi and the vital capacity of the patients was usually less than 31 . so that the magnification error on full inspiration is probably much less than $5 \%$. All measurements were corrected to give a mean magnification of 3 .

The procedure used in the investigations was as follows. After the routine films of the bronchogram had been taken the patient was encouraged to suppress coughing, and an oesophageal balloon attached to the end of a polyethylene tube was passed until the balloon lay in the lower third of the oesophagus. The patient lay supine on the couch and the oesophageal tube was attached to a strain gauge for recording intra-oesophageal pressure. Respiratory volumes were recorded by the patient breathing into a closed-circuit spirometer system. A potentiometer attached to the wheel of the spirometer allowed volume changes together with the intra-oesophageal pressure to $x$ be recorded electrically on a three-channel direct- $\frac{\dot{\sigma}}{\partial}$ writing oscillograph. The third channel of the oscillograph recorded the instant at which the exposure was made.

Films were taken while the patient was carrying out various respiratory manœuvres, and at no time was breathing voluntarily arrested. The first film was $\rightarrow$ taken during quiet breathing at the end of an ordinary expiration (functional residual capacity or F.R.C.). N The patient was then instructed to take a slow, full inspiration and a second radiograph was taken at the $N$ height of the inspiration. A third was taken at the $\underset{\omega}{N}$ end of a full expiration. He was then instructed to 0 cough, and a fourth radiograph was taken at the instant of coughing.

The intra-oesophageal pressures were measured $\mathbb{\Phi}$ relative to the pressure at the end of a quiet expira-? tion. The bronchial diameters were measured directly ${ }^{0}$ from the film, and the bronchi selected for measurement were those which could be identified in all four $\stackrel{\Phi}{\Phi}$ films. When the diameter of the trachea has been $\mathbb{\mathbb { D }}$ measured this has been included in the group of large $O$ bronchi. Bronchial lengths were not measured because rotation of bronchi would give false impressions of 0 changes in length. 
T A B LE I

MEAN AND RANGE OF BRONCHIAL DIAMETERS, LUNG VOLUMES, AND INTRA-OESOPHAGEAL PRESSURES IN 10 SUBJECTS

\begin{tabular}{|c|c|c|c|c|c|c|}
\hline & \multirow{2}{*}{$\begin{array}{l}\text { Volume } \\
(l .)\end{array}$} & \multirow{2}{*}{$\begin{array}{l}\text { Intra-oesophageal } \\
\text { Pressure } 1 \\
\text { (cm.water) }\end{array}$} & \multicolumn{4}{|c|}{ Change in Bronchial Diameters (\%) } \\
\hline & & & $<1.7 \mathrm{~mm}^{2}$ & $1.7-3.5 \mathrm{~mm}$ & $3.5-7.0 \mathrm{~mm}$ & $>7.0 \mathrm{~mm}$. \\
\hline Full inspiration & $\begin{array}{l}+1.43 \\
(+0.89 /+2.46)^{3}\end{array}$ & $\begin{array}{l}-24 \cdot 2 \\
(-8 \cdot 5 /-43 \cdot 5)\end{array}$ & $\begin{array}{l}+28 \\
(+3 /+66)\end{array}$ & $\begin{array}{l}+14 \\
(-3 /+25)\end{array}$ & $\begin{array}{l}+15 \\
(+11 /+19)\end{array}$ & +5 \\
\hline Full expiration & $\begin{array}{l}-0.48 \\
(+0 \cdot 1 /-0.78)\end{array}$ & $\begin{array}{l}+14 \cdot 7 \\
(+2 \cdot 7 /+34 \cdot 0)\end{array}$ & $\begin{array}{l}-13 \\
(+3 /-26)\end{array}$ & $\begin{array}{l}-16 \\
(+5,-29)\end{array}$ & $\begin{array}{l}-10 \\
(+2 /-17)\end{array}$ & $\begin{array}{l}-7 \\
(-3 /-12)\end{array}$ \\
\hline Cough & $\begin{array}{l}+0.92 \\
(+0.47 /+1.48)\end{array}$ & $\begin{array}{l}+83 \\
(+34 /+143)\end{array}$ & $\stackrel{+1}{(+15 /-9)}$ & $\begin{array}{l}-6 \\
(+7 /-16)\end{array}$ & $\begin{array}{l}-15 \\
(+1 !-58)\end{array}$ & $\begin{array}{l}-15 \\
(+4 /-41)\end{array}$ \\
\hline $\begin{array}{l}\text { No. of bronchi } \\
\text { measured }\end{array}$ & & & 29 & 13 & 8 & 9 \\
\hline
\end{tabular}

\section{RESULTS}

In this article the use of the term 'airways' or 'bronchi' is intended to include the trachea, bronchi, and bronchioli. Airways of $7.0 \mathrm{~mm}$. or over in diameter correspond to the trachea and the main and lobar bronchi, those of 3.5 to 7.0 $\mathrm{mm}$. include bronchi down to about their third division. Airways of less than $1.7 \mathrm{~mm}$. correspond to the smallest bronchi and bronchioli, though where one finishes and the other begins cannot be determined radiologically. In this series the smallest airway measured was $0.6 \mathrm{~mm}$. in diameter at the functional residual capacity.

Measurements were made on a total of 59 bronchi in the 10 patients studied. The percentage change in diameter, relative to that at the F.R.C., at full inspiration, at full expiration, and during coughing, is shown in Table $I$. The range of values for bronchial diameters shown in the table is the range of the mean value for each subject. In each subject the enlargement of the individual bronchi on inspiration was usually of the same order. For example, for the smaller airways it was from 12 to $50 \%$ in one subject and from 50 to $104 \%$ in another, but occasional bronchi did appear to decrease slightly in diameter whilst the majority increased.

At the height of an inspiration the mean diameter of all the airways increased but the percentage increase was greater in the smaller airways, being $28 \%$ in the airways of less than 1.7 $\mathrm{mm}$. diameter and only $5 \%$ in those over $7 \mathrm{~mm}$. diameter. On expiration the bronchi decreased in diameter but the response in the bronchi of different sizes was less variable than on inspiration. The intra-oesophageal pressure recorded on full expiration in these supine subjects is probably higher than the intrathoracic pressure (Knowles, Hong, and Rahn, 1959).

On coughing high intrathoracic pressures were recorded at the instant that the radiograph was taken (Table I), but in spite of these the bronchial narrowing was hardly greater than on full expiration. In some instances there was a greater bronchial diameter on coughing than there was at the F.R.C. Compression of the trachea or major bronchi to approximately half the initial diameter was seen in a few of the cough films. Figure 1 shows the bronchograms for one patient. Changes in calibre and irregularities in the larger bronchi are to be noted. Close examination of the smaller bronchi and bronchioli, as indicated, shows that at the instant of coughing the diameters approximate to those seen at rest (F.R.C.) and are greater than during expiration.

\section{DISCUSSION}

Bronchial and tracheal diameters may be altered either by a change in their transmural pressure or by the action of smooth muscle in the bronchial walls. In these studies the films during inspiration and expiration were taken when the flow of air was almost zero, that is, when the intrabronchial pressure was atmospheric. Under static conditions of inspiration and expiration the changes in transmural pressure of the trachea and large bronchi can be taken to be the same as the changes in intra-oesophageal pressure because when the airways are not closely surrounded by alveolar tissue the pressure outside the wall of the bronchus is probably the same as the intrathoracic pressure. The smaller bronchi, which have alveoli applied to their outer surfaces, have an extramural pressure which cannot be calculated simply.

The changes in bronchial diameter obtained in the patients studied were similar to those reported by Stutz (1950a) in eight patients. The magnitude of the change in diameter, relative to that at the F.R.C., on full inspiration was very similar to that on full expiration in spite of the volume expired being much smaller than that which was inspired. The greater change in bronchial diameter per unit change in lung volume below 

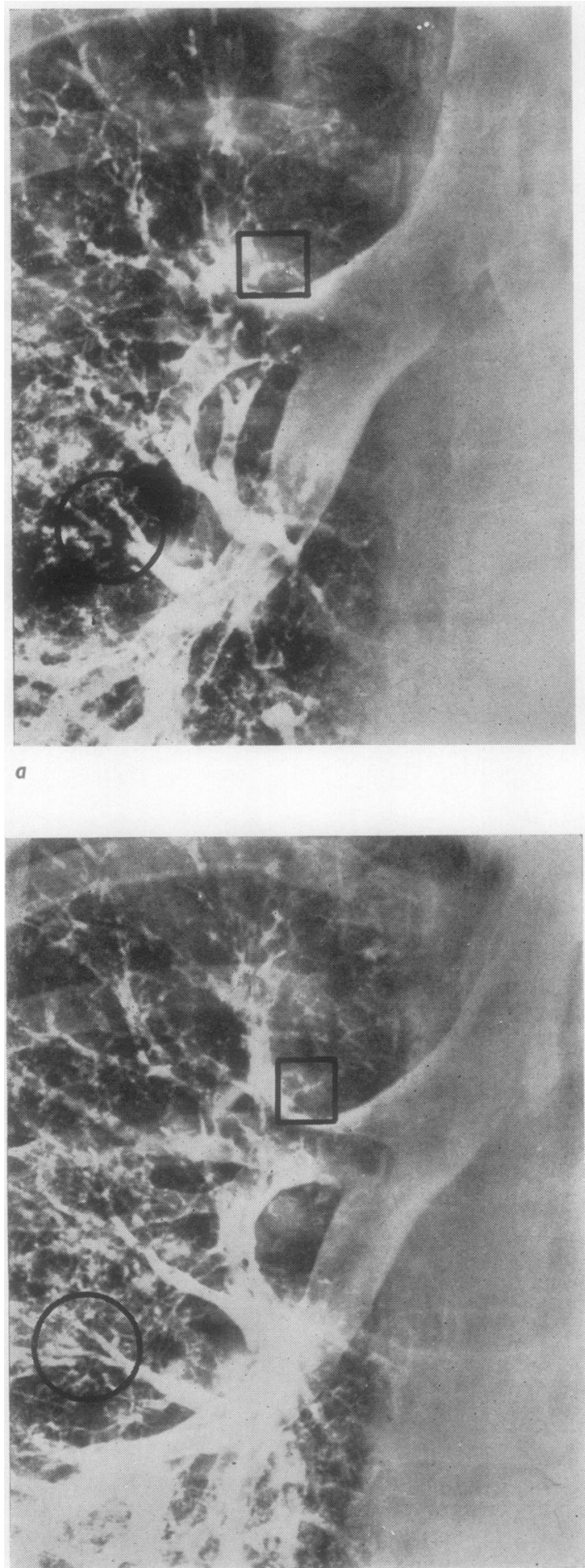

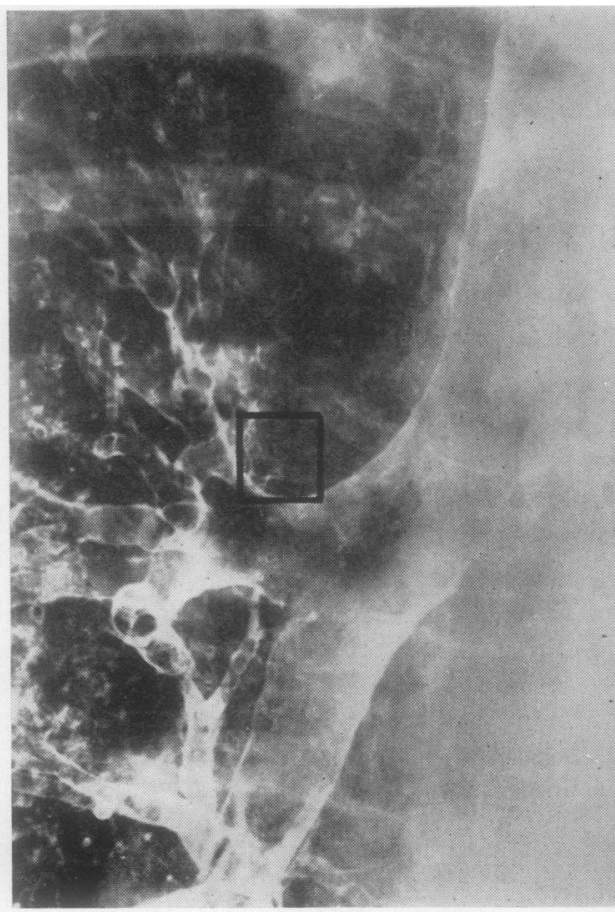

b



FIG. 1. Bronchograms (a) at the F.R.C., (b) at full inspiration, (c) at full expiration, and (d) during coughing. Radiographs reproduced at half the original size. Corresponding airways are shown by the square and the circles 
the F.R.C. is similar to that observed in isolated dog lungs (Marshall, 1962). All the changes in bronchial diameters which were measured could have been due to changes in lung volume or in intrathoracic pressure, and there was no reason to postulate active contraction of smooth muscle in the bronchial wall.

If one assumes that the air flow in the bronchi is linear and obeys Poiseuille's law that the resistance to flow is proportional to the length and to the reciprocal of the fourth power of the radius, then a $28 \%$ change in radius, such as might occur in a bronchus $1 \mathrm{~mm}$. in diameter, will change the resistance by $60 \%$, whereas a $5 \%$ change, which occurs in the larger bronchi, will change the resistance by only $18 \%$. Airways of less than $3 \mathrm{~mm}$. diameter probably account for $90 \%$ of the resistance of the whole bronchial tree during quiet breathing (Rohrer, 1915), so it is probable that the change in resistance that accompanies changes in lung volume (Briscoe and DuBois, 1958) is due mainly to a change in diameter of the smaller airways.

Stutz (1950b) found that on coughing the bronchial diameters were less than half the diameters on expiration and less than one third the diameters on inspiration. The change was greater in the large and middle-sized bronchi and less in the smaller bronchi. No measurements were made of the lung volume at which the cough occurred or of the pressures developed during the cough. The present study showed no consistent compression of the smaller bronchi during coughing in spite of the high intrathoracic pressures; in fact there was a small increase $(+1 \%)$ in the mean diameter of the smaller airways relative to their diameter at the F.R.C. The cough radiographs were taken at a mean lung volume of 0.92 litre above the F.R.C. and during a normal inspiration to this volume the diameter of the small airways would be expected to be increased about $20 \%$ above that at the F.R.C. During cough, therefore, there is evidence of compression of all sizes of bronchi but if the cough takes place at an increased lung volume the distending effect of the lung parenchyma protects the bronchi, particularly the smaller ones, from compression.

In addition to the distending effect of the lung parenchyma the smaller bronchi are less liable to collapse during cough because, being the site of airway resistance, the pressure in the lumen of the bronchi is much higher than that in the trachea and larger bronchi. The marked collapse of the trachea and larger bronchi, which occurs during coughing, aids the effectiveness of the cough by increasing the air velocity (Ross et al., 1955). It is fortunate that during coughing the narrowing is more pronounced in the medium and larger bronchi where coughing is the principal mechanism for the removal of mucus. In the smaller bronchi the velocity of air flow is very much less than in the trachea (Rohrer, 1915) and ciliary action is much more important.

If the narrowing were to occur principally in the smaller airways of less than $3 \mathrm{~mm}$. diameter, which normally provide most of the resistance to breathing, the resistance to breathing would be increased so much and the air velocity would be so reduced that the coughing would be ineffective. For example, if the resistance to air flow in the bronchial tree of a subject is $2 \mathrm{~cm}$. water $/ 1$. $/ \mathrm{sec}$. and if $90 \%$ of the resistance is in the smaller bronchi, the resistance of these small bronchi will be $1.8 \mathrm{~cm}$. water $/ 1 . / \mathrm{sec}$. and that of the larger bronchi $0.2 \mathrm{~cm}$. water $/ 1 . / \mathrm{sec}$. If the diameter of the smaller bronchi were reduced to one half during coughing the resistance would increase by 16 times, assuming for the sake of ease of calculation that the air flow remains laminar, to $28 \cdot 8 \mathrm{~cm}$. water $/ 1$. $/ \mathrm{sec}$. If the diameter of the larger bronchi were reduced by half their resistance would increase by 16 times to $3 \cdot 2 \mathrm{~cm}$. water $/ 1$. $/ \mathrm{sec}$. Thus if the smaller bronchi were narrowed to half their diameter an intrathoracic pressure of $100 \mathrm{~cm}$. water would produce an air flow of only 3.3 1./sec. $(100 / 28 \cdot 8+0 \cdot 2)$. If only the large bronchi were reduced to half their diameter an intrathoracic pressure of $100 \mathrm{~cm}$. water would produce an air flow of $201 . / \mathrm{sec}$. $(100 / 1 \cdot 8+3 \cdot 2)$. At small lung volumes compression of the smaller bronchi can occur during coughing and this is probably the reason why coughing at small lung volumes is so ineffective.

Although the small bronchi are not compressed during coughing, neither are they distended, and the results reported here do not support the theory that in a normal lung coughing can lead to destruction and breakdown of the smaller airways and alveoli. In a diseased lung, where some bronchi are narrowed, it is possible that during coughing distension of some lung units may occur by the mechanism suggested by Rohrer (1921).

The extent to which normal bronchi may appear irregular and deformed is as yet impossible to define. At all times the outlines of the walls of normal bronchi are never parallel, nor do they ever appear as smooth, elongated cylindrical tubes. This can be seen by holding the edge of a ruler against a contrast-coated bronchus. Using the magnification technique described, this becomes more manifest for it is in the smaller airways that these linear changes are more marked. Minor 
ripples, undulations, and irregularities are normally to be expected, associated with such things as the presence of mucus, with contractions of smooth muscle and alterations in the tone of the bronchial walls, together with the limiting effect of the bronchial cartilages. However, the extent to which these irregularities of outline occur in bronchi without apparent disease can be considerable. Alterations have also been observed in bronchi where narrowing was seen to occur during expiration and coughing. This was often more
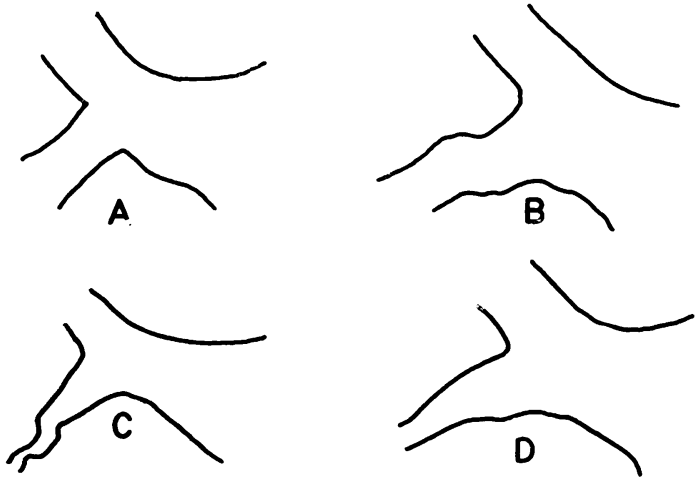

$1 \mathrm{~cm}$.

FIG. 2. Tracings of a section of the bronchial tree (A) at the F.R.C., (B) at full inspiration, (C) at full expiration, and (D) during coughing.

marked during expiration than during coughing, in spite of the high intrathoracic pressures developed during coughing (Fig. 2). The bronchial irregularities seen during full inspiration are usually not so marked, which in part is explained by the limiting effect of the bronchial cartilages.

\section{SUMMARY}

After completing routine bronchography a magnified film technique was used to study the diameters of the small bronchi at resting lung volume, and during inspiration, expiration, and coughing. Lung volume and intra-oesophageal pressure were measured simultaneously.

Bronchial diameters increased on full inspiration and decreased on full expiration ; the changes were more marked in the smaller bronchi.

On coughing the compression was more marked in the larger bronchi, and the increased lung volume at which coughing occurred appeared to be important in maintaining the diameter of the smaller bronchi. The significance of the site of bronchial compression in relation to the effectiveness of coughing is discussed.

We would like to express our thanks to our radiological and clinical colleagues for their co-operation and help, and in particular to Miss E. Emrys-Roberts for her technical assistance and support.

\section{REFERENCES}

Briscoe, W. A., and DuBois, A. B. (1958). J. clin. Invest., 37, 1279. Dekker, E.. and Groen, J. (1957). Lancet, 1, 1064.

Holden, W. S., and Ardran, G. M. (1957). J. Fac. Radiol. (Lond.) $8,267$.

Knowles, J. H., Hong, S. K., and Rahn, H. (1959). J. appl. Physiol., 14,525 .

Marshall, R. (1962). Ibid., 17, 596.

Rohrer, F. (1915). Pflügers Arch. ges. Physiol., 162, 225.

- (1921). Schweiz, med. Wschr., 2, 741.

Ross, B. B., Gramiak, R., and Rahn, H. (1955). J. appl. Physiol., $8,264$.

Stutz, E. (1950a). Fortschr. Röntgenstr., 72, 309.

(1950b). Ibid., 72, 447. 